\title{
Improvement of WLAN Contention Resolution by Loss Differentiation
}

\author{
(TW04-712, Final Version) \\ Qixiang Pang ${ }^{1}$, Victor C. M. Leung ${ }^{1}$, Fellow, IEEE, Soung C. Liew ${ }^{2}$, Senior Member, IEEE \\ \{qixiangp@ece.ubc.ca, vleung@ece.ubc.ca, soung@ie.cuhk.edu.hk\} \\ 1. Department of Electrical and Computer Engineering \\ The University of British Columbia \\ 2. Department of Information Engineering \\ The Chinese University of Hong Kong
}

\begin{abstract}
In a realistic WLAN environment, frame losses may be caused by collisions or channel noise. The existence of noise-induced losses reduces the effectiveness of the standard WLAN backoff algorithm for contention resolution, which assumes that all losses are caused by collisions and always doubles the contention window to reduce contention upon a frame loss. In this paper, we propose new backoff algorithms that take advantage of a new capability to differentiate the losses, and thereby sharpen the accuracy of the contention resolution process. Analytical models are developed to analyze the performance of these algorithms under heterogeneous link conditions in a WLAN. Both analysis and simulation results show that significant improvement of throughput and fairness can be obtained for WLANs in which contention resolution is enhanced by the loss differentiation ability.
\end{abstract}

Index Terms - WLAN, MAC Protocol, Contention Resolution, Backoff Algorithm, Loss

Differentiation

Correspondence to: Qixiang Pang

Email: qixiangp@ece.ubc.ca; Tel: +1-604-8224985

2332 Main Mall, Vancouver, BC, V6T 1Z4, CANADA

This work was sponsored by the Canadian Natural Sciences and Engineering Research Council under grant STPGP 257684-02 and the AoE scheme under the University Grant Committee of Hong Kong (AoE/E01/99). 


\section{INTRODUCTION}

The use of IEEE 802.11 wireless local area networks (WLANs) has been spreading quickly [1]. All 802.11 WLAN devices use the Distributed Coordination Function (DCF) to coordinate channel access by means of carrier sense multiple access with collision avoidance (CSMA/CA). In DCF, after sending a data frame, the sending station considers the frame to be lost if it does not receive the acknowledgment (ACK) frame, and retransmits the data frame after a random delay given by the binary slotted exponential backoff algorithm [1].

The standard backoff algorithm in DCF serves the purpose of contention resolution. It assumes that all frame losses are due to collisions (referred as collision losses) and does not take the possibility of transmission errors due to noise and interference into consideration. When the channel condition is not ideal, a data frame can be corrupted by random transmission errors, resulting in the receiver not returning an ACK frame to the sender (referred as a transmission loss). In the standard algorithm, since the sender attempts to reduce contention by doubling the maximum backoff time whenever it experiences frame losses, the backoff algorithm will cause unnecessarily long delay and poor channel utilization when noisy channel conditions cause successive transmission losses. This problem exists in all other known backoff algorithms [2][3][4] as well.

In a realistic WLAN environment, both types of losses mentioned above can occur. Without differentiating the possible causes of each frame loss, the DCF cannot adjust the backoff process appropriately, resulting in poor performance under noisy channel conditions. To solve this problem, we propose in this paper to apply recently developed loss differentiation (LD) methods [5] to the standard backoff algorithm, as well as several new variants of it. Analytical models of the backoff algorithms are established for performance evaluation. One of the new backoff algorithms is recommended because it exhibits the best performance overall. Analytical and simulation results show that the new backoff algorithm enhanced with LD capability significantly improve throughput and fairness in WLANs with multiple stations under heterogeneous channel conditions, as it adapts 
more accurately to the contention and channel error conditions experienced by each station.

The organization of the remainder of this paper is as follows. Section II reviews the problem in the existing backoff algorithm for contention resolution. New backoff algorithms based on LD capability are proposed. In Section III, the performance of the new algorithms is analyzed and compared with that of the standard algorithm. Section IV concludes this paper.

\section{NEW WLAN PRotocol WITH LOSS DIFFERENTIATION}

\section{A. Problem with the Existing Backoff Algorithms in WLAN}

In IEEE 802.11 DCF [1], when a station has a data frame to transmit, a random backoff time (in unit of timeslot) is selected uniformly between 0 and $c w-1$, where $c w$ is the so-called contention window. If this is a new frame transmitted for the first time, $c w$ is set to CWmin, the minimum (initial) contention window. Once the backoff time is chosen, the station must wait until the backoff timer reaches zero before it can transmit the frame. If the station does not receive an ACK frame after sending the data frame, it assumes that a collision has occurred, doubles $c w$, and restarts the countdown with a random backoff time based on this larger $c w$ value before retransmitting the data. The rationale is that if a collision happens, the contention intensity must be higher than expected orginally, which calls for a larger $c w$ to lower the rate of retransmission attempts for better contention resolution.

However, in a noisy wireless environment, unsuccessful reception of the data frame can also be caused by channel noise or other interference, e.g., from a Bluetooth device. The assumption that all frame losses are due to collisions between WLAN devices is generally not true. When a data frame is received and decoded at the receiver, if the Frame Check Sequence (FCS) does not check out due to a few bit errors in the frame, the receiver silently discards the frame and the sender will not receive an ACK frame. The standard backoff algorithm in this case will cause the sender to inappropriately perform contention resolution by doubling its contention window for the next 
retransmission attempt. Consequently, the performance of the system could become degraded unless the standard backoff algorithm is enhanced with loss differentiation capability.

Several alternate backoff algorithms [2][3][4] have been proposed to improve the contention resolution performance of the standard backoff algorithm. The major difference from the standard algorithm is that in these algorithms, when $c w$ exceeds $C W m i n, c w$ is not reset to $C W m i n$ when the transmission is successful or the number of retransmissions reaches its limit. Instead, $c w$ is either halved [2][4] or reduced by a factor of $1 / \sqrt{2}$ [3]. The rationale is that a large $c w$ value suggests intensive contention, which calls for maintaining $c w$ at a high level or slowing down the reduction of $c w$ to $C W m i n$. When there are many stations contending for channel access, it has been shown in [2][3][4] that these algorithms provide better performance than the standard backoff algorithm. However, this is true only when the transmissions in a WLAN are reliable and all frame losses are caused by collisions. When transmission losses occur, $c w$ can also reach a high value. If $c w$ is maintained at a high level, more air time can be wasted than in the standard algorithm. Without LD capability, the performance of these algorithms can be even worse than the standard algorithm.

\section{B. Loss Differentiation Methods in WLAN}

The LD methods for WLAN have been proposed in the preliminary work [5]. However, this work lacks a detailed performance evaluation, nor has its application to improve WLAN contention resolution been considered thoroughly. Here we explain how the basic and RTS/CTS access procedures in the 802.11 DCF [1] can be enhanced with LD capability as proposed in [5].

The RTS/CTS access is a 4-way handshake procedure. In RTS/CTS access, before a data frame transmission, the sender sends an RTS (Request-To-Send) frame and the receiver returns a CTS (Clear-To-Send) frame to the sender upon receiving the RTS. Successful exchange of RTS/CTS clears the channel and reserves it for the sender's data transmission. RTS and CTS are short control frames. Since CTS and ACK frames provide feedback from the intended receiver, the sender can utilize them for LD purposes as follows: (i) If both the CTS and then the subsequent ACK frames are received, the data frame transmission is successful. (ii) If the CTS frame is 
received but the ACK frame is not, the data frame transmission has failed, most likely due to channel noise. (iii) If the CTS frame is not received, most likely a collision has occurred. The 4-way message exchange sequence in RTS/CTS access is not changed.

Basic access is the default access method in DCF and employs a two-way handshake procedure. LD for basic access is not straightforward as only ACK frames provide feedback from receivers. The following describes a LD method for basic access which requires minimal modifications to the standard to provide additional feedback.

The LD method for basic access is based on the following observation. The data frame can be functionally partitioned into two parts: header and body. The MAC header contains information such as frame type, source address and destination address, and comes before the MAC body, which contains the data payload. In a WLAN with multiple stations sharing a common channel, a collision occurs when two or more stations start transmitting in the same time slot, which will likely corrupt the whole frame (header plus body) at the receiver. On the other hand, a frame transmission that is not affected by collision with transmission from another WLAN station may still be corrupted by noise and interference. However, under the condition that the signal-to-noise-plus-interference ratio (SINR) is reasonable to sustain a connection between the sending and receiving stations, the receiver is likely able to acquire the frame and decode it, as the physical header is transmitted at the base data rate for robustness (e.g., in $802.11 \mathrm{~b}$, the typical data rate is $11 \mathrm{Mbps}$, but the 192-bit physical header is always transmitted at $1 \mathrm{Mbps})$. In this case, the noise or interference may result in a few bit-errors that cause an FCS error in the decoded data frame, which is then discarded by the receiver. As the MAC header (18-30 bytes) is typically much shorter than the MAC body (e.g., a typical Internet Protocol datagram is several hundreds to a couple of thousands bytes long), when FCS fails, it is much more likely caused by bit errors in the body than the header.

If the MAC header is correctly received but the body is corrupted, the receiver can observe the MAC header content to learn the identity of the sender and to verify that it is the intended receiver. To verify the correctness of the MAC header, a short Header Error Check (HEC) field can 
be added at the end of the header (Figure 1) to provide error checking over the header, while the FCS at the end of the frame provides error checking over the entire MAC frame. Note that the use of HEC in the header is not a new concept as it has been adopted in many other communication systems, such as asynchronous transfer mode (ATM) [8], Bluetooth [9] and WiMedia [10], all of which includes a 1-byte HEC or header check sequence (HCS) field in their header. With the HEC, when a data frame is received and FCS fails, the HEC can be verified to see if the header is free of error, and if so, proper feedback can be returned to the sender identified by the MAC header.

As discussed above, FCS failure but correct HEC in a frame reception is a good indication that the frame has been corrupted by transmission errors rather than a collision. Because in the original basic access, only ACK frames are available to provide positive feedback, a new control frame - NAK needs to be introduced to inform the sender that the data frame transmission has failed and the failure is due to transmission errors; i.e., the data frame has suffered a transmission loss. On the other hand, if the sender receives neither a NAK nor an ACK after sending a data frame, it is a good indication that the frame transmission has suffered a collision loss. The NAK frame can be implemented with exactly the same structure as the ACK frame except for a one-bit difference in the frame type field in the header, and is sent at the same data rate as an ACK frame. The transmission of a NAK does not consume more bandwidth or collide with other frames because it is transmitted SIFS after the data frame transmission and occupies the time that would have been used by the transmission of an ACK. The flow charts of the sender and receiver's operations in the new basic access are given in Figure 2.

The HEC field is a necessary modification to the standard because without it, when the FCS fails, the receiver would not be able to determine if the header is in error and would not be able to trust the sender address in the header for returning the NAK. The HEC field (which can be 1 or 2 bytes) costs an extra overhead. But it can be calculated that the overhead due to the extra field to the total transmission time is much less than 1\% [5]. Therefore the overhead is negligible. 
Comparing the two LD methods, RTS/CTS access is useful when the data frame size or the number of stations is very large or there are hidden terminals. However, as it consumes extra time for RTS/CTS exchange, RTS/CTS access is less efficient than basic access in other cases. The LD method for RTS/CTS access does not involve any modification to the standard. The LD method for basic access, however, needs two minor modifications to the current standard: the HEC field and the NAK frame, which are both easy to implement.

\section{New Backoff Algorithms Using Loss Differentiation}

When the causes of the frame losses can be differentiated, it will be beneficial for the sender to apply different backoff times for different types of losses. There are three possible cases for a frame transmission: (1) a collision occurs; (2) there are random bit errors in the frame due to noise; and, (3) the transmission is successful.

When a collision occurs (Case-1), contention resolution is required, and the contention window is doubled as in the standard. By doubling the backoff time of the collided stations, further collisions can be minimized.

When there are bit errors in the frame due to noise (Case-2), instead of doubling the contention window as in the standard, the station can choose to retransmit the data sooner by using a shorter backoff time than the standard, e.g., by keeping the contention window unchanged.

If the contention window has reached a high level due to collisions, the larger contention window implies that many stations are trying to send data and the contention intensity is high. When a transmission is successful (Case-3), instead of always resetting the contention window to CWmin for the transmission of the next data frame as in the standard, a different option is to halve the contention window. The larger one between the halved $c w$ and $C W m i n$ is chosen to be the new contention window. The purpose of keeping the contention window at a relatively high level is to avoid more collisions in the future in anticipation of a high degree of contention.

Based on the different combinations of the above options, we have four backoff schemes listed in Table 1. Among the four, Backoff-1 and Backoff-2 do not differentiate the two types of 
losses, whereas Backoff-3 and Backoff-4 do. Backoff-1 is just the standard algorithm defined in IEEE 802.11 [1]. Similar algorithms to Backoff-2 also have been studied in [2][3][4] for error-free WLAN links. Backoff-3 and Backoff-4 are the two new backoff algorithms proposed in this paper.

For Case-2 in Table 1, an Immediate Retry (IR) scheme can be introduced to possibly further enhance the WLAN performance together with the new backoff rules. The IR scheme borrows the concept of "burst" transmission which was first introduced in [11] and has now been incorporated into the 802.11e standard [12]. In the "burst" scheme, once a station obtains the transmission opportunity through contention, it can send more than one data frames. Here, in the IR scheme, for a station with loss differentiation ability, when a transmission loss is detected (Case-2), the station can retransmit immediately after a SIFS without a backoff. For basic access, the data frame is retransmitted immediately. For RTS/CTS access, there are two options: the station can either immediately retransmit an RTS to avoid hidden terminal, or retransmit the data frame only. A control parameter, maximum number of immediate retries, can be used to limit the maximum number of consecutive immediate retries when successive transmission losses occur.

\section{iII. Performance Evaluations}

\section{A. Effectiveness of the Loss Differentiation Methods}

Before we discuss the performance of the new backoff algorithms, we first evaluate the effectiveness the proposed LD methods in terms of the probability of successfully detecting a transmission loss. We stress that the LD methods are not intended to be totally reliable, but if they are successful in differentiating transmission losses from collision losses most of the time, then they give the basis for improving the performance of contention resolution as proposed in this paper.

For RTS/CTS access, when either the RTS or CTS frame is lost due to noise, the sender will misunderstand it as a collision loss. The probability of successful transmission loss detection is

$$
P_{D}^{R C T S}=\left(1-p_{e}^{R T S}\right)\left(1-p_{e}^{C T S}\right)
$$

where, $p_{e}^{R T S}$ and $p_{e}^{\text {CTS }}$ are the error rates of RTS and CTS frames. 
For basic access, when a data frame is in-error due to noise, in the following two cases, the sender will not be able to get a NAK correctly and thus misunderstand it as a collision loss: (i) the header of the data frame is erroneous and therefore the receiver does not generate a NAK; (ii) the header is correct and NAK is generated but the NAK frame is erroneous. When a data frame is successfully delivered but the corresponding ACK is lost, the sender will also misunderstand it as a collision loss. Therefore the probability of successful transmission loss detection is

$$
P_{D}^{\text {basic }}=1-\frac{\left(p_{e}^{H}+\left(1-p_{e}^{H}\right) \times p_{e}^{B} \times p_{e}^{N A K}\right)+\left(1-p_{e}^{D A T A}\right) \times p_{e}^{A C K}}{p_{e}^{D A T A}+\left(1-p_{e}^{D A T A}\right) \times p_{e}^{A C K}}
$$

where, $p_{e}^{A C K}, p_{e}^{N A K}, p_{e}^{D A T A}, p_{e}^{H}$ and $p_{e}^{B}$ are the error rates of ACK, NAK, data frame, data frame header, and data frame body, respectively.

\section{B. Analytical Modeling of the Backoff Algorithms}

The performance of standard backoff algorithm (Backoff-1) in a WLAN has been analyzed in [13] [14], and algorithms similar to Backoff-2 have been analyzed in [2][3][4]. However, these analyses do not take channel noise into consideration. The performance of the standard backoff algorithm subject to link errors with a homogeneous error rate has been analyzed in [15][16][17]. However, none of the prior work has considered heterogeneous link conditions or control frame errors, and other backoff algorithms have not been evaluated with frame errors. The analysis becomes more complex when these realistic conditions are considered for the newly proposed backoff algorithms,

We consider a WLAN in which there are multiple active stations and the wireless links are prone to transmission losses due to the existence of noise or interference. Each active station generates a contending traffic flow and the stations always have frames to send. All the four algorithms working over the noisy links have been modeled; however, due to space limitation, only the analytical procedures of Backoff- 1 and Backoff- 4 are presented here.

Consider $L$ types of noisy links with different SINR levels. Let $p_{l, e}^{R T S}, p_{l, e}^{C T S}, p_{l, e}^{A C K}, p_{l, e}^{N A K}$, $p_{l, e}^{D A T A}, p_{l, e}^{H}$, and $p_{l, e}^{B}$ represent the RTS error rate, CTS error rate, ACK error rate, NAK error rate, 
data frame error rate, header error rate of a data frame, and body error rate of a data frame, respectively, over a type $l(1 \leq l \leq L)$ link. Further, let $T_{\text {SIFS }}, T_{D I F S}, T_{A C K}, T_{N A K}, T_{R T S}, T_{C T S}$ and $T_{\text {DATA }}$ represent the transmission times of SIFS, DIFS, ACK, NAK, RTS, CTS, and data frames, respectively.

We let $p_{l, c}$ denote the collision probability of a data or RTS frame transmitted over a type $l$ $(1 \leq l \leq L)$ link, which is assumed to be constant and independent of the backoff stage of the frame. This probability varies across different link types as different loss rates result in variations in average contention window size. Let $s_{l}(t)$ be the random process representing the backoff stage at time $t$ for a type $l$ link. Let $b_{l}(t)$ be the random process representing the backoff counter during a particular backoff stage. The state variables $\left\{s_{l}(t), b_{l}(t)\right\}$ define a two-dimensional discrete-time Markov chain. For brevity, we adopt the short notation:

$$
P_{l}\left\{i_{1}, k_{1} \mid i_{0}, k_{0}\right\}=P\left\{s_{l}(t+1)=i_{1}, b_{l}(t+1)=k_{1} \mid s_{l}(t)=i_{0}, b_{l}(t)=k_{0}\right\}
$$

Also, define $b_{l, i, k}=\lim _{t \rightarrow \infty} \operatorname{Pr}\left\{s_{l}(t)=i, b_{l}(t)=k\right\}$ to be the stationary distribution of the Markov chain for a type $l$ link. Other notations used in the analysis are as follows:

$N_{l}$ : the number of active stations operating over a type $l$ link;

$\tau_{l}$ : the probability of a station transmitting during a slot time over a type $l$ link;

$m$ : the maximum backoff stage beyond which the window size will be kept constant;

$W_{i}$ : the window size at backoff stage $i, 0 \leq i \leq m$.

\section{1). Backoff-1 Algorithm for Heterogeneous Links}

The state transition diagram of the standard backoff algorithm (Backoff-1) is given in Figure 3. The only non-null one-step transition probabilities in the Markov chain are as follows:

$$
\begin{cases}P_{l}\{i, k \mid i, k+1\}=1 & 0 \leq i \leq m, 0 \leq k \leq W_{i}-2 \\ P_{l}\{0, k \mid i, 0\}=\left(1-p_{l}\right) / W_{0} & 0 \leq i \leq m, 0 \leq k \leq W_{0}-1 \\ P_{l}\{i, k \mid i-1,0\}=p_{l} / W_{i} & 1 \leq i \leq m, 0 \leq k \leq W_{i}-1 \\ P_{l}\{m, k \mid m, 0\}=p_{l} / W_{m} & 0 \leq k \leq W_{m}-1\end{cases}
$$


where $p_{l}$ is the probability of an unsuccessful transmission due to either a collision or bit errors in the frame. For basic access,

$$
p_{l}=p_{l}^{\text {basic }}=1-\left(1-p_{l, c}\right)\left(1-p_{l, e}^{\text {DATA }}\right)\left(1-p_{l, e}^{A C K}\right)
$$

For RTS/CTS access,

$$
p_{l}=p_{l}^{R C T S}=1-\left(1-p_{l, c}\right)\left(1-p_{l, e}^{R T S}\right)\left(1-p_{l, e}^{C T S}\right)\left(1-p_{l, e}^{D A T A}\right)\left(1-p_{l, e}^{A C K}\right)
$$

The relations among the stationary distributions of the Markov chain states are

$$
\begin{cases}b_{l, m, k}=\frac{W_{m}-k}{W_{m}} \times\left(b_{l, m, 0}+b_{l, m-1,0}\right) \cdot p_{l} & 0 \leq k \leq W_{m}-1 \\ b_{l, i, k}=\frac{W_{i}-k}{W_{i}} \times\left(b_{l, i-1,0} \cdot p_{l}\right) & 1 \leq i \leq m-1,0 \leq k \leq W_{i}-1 \\ b_{l, 0, k}=\frac{W_{0}-k}{W_{0}} \times\left(\sum_{i=0}^{m} b_{l, i, 0} \cdot\left(1-p_{l}\right)\right) & 0 \leq k \leq W_{0}-1\end{cases}
$$

Based on (7), all $b_{l, i, 0}(1 \leq i \leq m)$ can be expressed as functions of $b_{l, 0,0}$ as follows:

$$
b_{l, i, 0}=b_{l, 0,0} \cdot p_{l}{ }^{i}, \quad 1 \leq i \leq m-1
$$

and,

$$
b_{l, m, 0}=\frac{p_{l}{ }^{m}}{1-p_{l}} b_{l, 0,0}
$$

Summing (7) over $k$, we have

$$
\left\{\begin{array}{l}
\sum_{k=0}^{W_{m}-1} b_{l, m, k}=\frac{W_{m}+1}{2} \times\left(b_{l, m, 0}+b_{l, m-1,0}\right) \cdot p_{l} \\
\sum_{k=0}^{W_{i}-1} b_{l, i, k}=\frac{W_{i}+1}{2} \times\left(b_{l, i-1,0} \cdot p_{l}\right) \quad 1 \leq i \leq m-1 \\
\sum_{k=0}^{W_{0}-1} b_{l, 0, k}=\frac{W_{0}+1}{2} \times\left(\sum_{i=0}^{m} b_{l, i, 0} \cdot\left(1-p_{l}\right)\right)
\end{array}\right.
$$

Now, the normalization condition for stationary probability distribution requires

$$
\sum_{i=0}^{m} \sum_{k=0}^{W_{i}-1} b_{l, i, k}=1
$$


By solving $b_{l, 0,0}$ from (11) based on (8), (9) and (10), $b_{l, 0,0}$ can be expressed as a function of $m$, $W_{i}(0 \leq i \leq m)$ and $p_{l}$.

Also note that $\tau_{l}$ is expressed by

$$
\tau_{l}=\sum_{i=0}^{m} b_{l, i, 0}
$$

and $p_{l, c}$ by

$$
p_{l, c}=1-\prod_{j=1}^{l-1}\left(1-\tau_{j}\right)^{N_{j}} \cdot\left(1-\tau_{l}\right)^{N_{l}-1} \cdot \prod_{j=l+1}^{L}\left(1-\tau_{j}\right)^{N_{j}}
$$

Finally, using the above equations, $b_{l, 0,0}, p_{l, c}$, and $\tau_{l}$ can be solved numerically. Then the total throughput over link type $l$ measured by MAC layer payload, $S_{l}$, is given by

$$
S_{l}=\frac{p_{l, s} p_{t r} D}{\left(1-p_{t r}\right) \sigma+p_{t r} T}
$$

where,

$D$ is the data frame payload size;

$\sigma$ is the duration of an empty slot time;

$p_{t r}$ is the probability that there is at least one transmission in a considered slot time,

$$
p_{t r}=1-\prod_{j=1}^{L}\left(1-\tau_{j}\right)^{N_{j}}
$$

$p_{l, s}$ is the probability that a transmission occurring on link type $l$ is successful given that there is at least one transmission in the considered slot time;

$T$ is the average time sensed busy when there is at least one transmission;

$p_{l, s}$ and $T$ depend on the access types. By considering all data frame and control frame transmission possibilities, the derivations of $p_{l, s}$ and $T$ are as follows.

For basic access, we have 


$$
\begin{aligned}
& p_{l, s}=p_{l, s}^{\text {basic }}=\left(1-p_{l, e}^{D A T A}\right) \cdot\left(1-p_{l, e}^{A C K}\right) \cdot p_{l, g} \\
& T=T^{\text {basic }}=T_{D I F S}+T_{D A T A}+T_{S I F S}+T_{A C K}
\end{aligned}
$$

where

$$
p_{l, g}=\prod_{j=1}^{l-1}\left(1-\tau_{j}\right)^{N_{j}} \cdot N_{l} \tau_{l}\left(1-\tau_{l}\right)^{N_{l}-1} \cdot \prod_{j=l+1}^{L}\left(1-\tau_{j}\right)^{N_{j}} / p_{t r}
$$

is the probability that a type $l$ station successfully obtains the transmission opportunity by contention given that there is at least one transmission in the considered slot time.

For RTS/CTS access, we have

$$
\begin{aligned}
& p_{l, s}=p_{l, s}^{R C T S}=\left(1-p_{l, e}^{R T S}\right)\left(1-p_{l, e}^{C T S}\right)\left(1-p_{l, e}^{D A T A}\right)\left(1-p_{l, e}^{A C K}\right) \cdot p_{l, g} \\
& T=T^{R C T S}= \\
& \sum_{l=1}^{L} p_{l, g} \cdot\left(\left(1-p_{l, e}^{R T S}\right)\left(1-p_{l, e}^{C T S}\right) \cdot T_{l o n g}+\left(1-\left(1-p_{l, e}^{R T S}\right)\left(1-p_{l, e}^{C T S}\right)\right) \cdot T_{\text {short }}\right)+\left(1-\sum_{l=1}^{L} p_{l, g}\right) \cdot T_{\text {short }}
\end{aligned}
$$

where,

$$
\begin{aligned}
& T_{\text {long }}=T_{\text {DIFS }}+T_{\text {RTS }}+T_{\text {SIFS }}+T_{\text {CTS }}+T_{\text {SIFS }}+T_{\text {DATA }}+T_{\text {SIFS }}+T_{A C K} \\
& T_{\text {short }}=T_{\text {DIFS }}+T_{R T S}+T_{\text {SIFS }}+T_{C T S}
\end{aligned}
$$

\section{2). Backoff-4 Algorithm for Heterogeneous Links}

The state transition diagram of Backoff-4 algorithm is given in Figure 4. The only non-null one-step transition probabilities in the Markov chain are as follows:

$$
\begin{cases}P_{l}\{i, k \mid i, k+1\}=1 & 0 \leq i \leq m, 0 \leq k \leq W_{i}-2 \\ P_{l}\{i, k \mid i+1,0\}=\left(1-p_{l, 1}-p_{l, 2}\right) / W_{i} & 0 \leq i \leq m-1,0 \leq k \leq W_{i}-1 \\ P_{l}\{i, k \mid i-1,0\}=p_{l, 1} / W_{i} & 1 \leq i \leq m, 0 \leq k \leq W_{i}-1 \\ P_{l}\{0, k \mid 0,0\}=\left(1-p_{l, 1}\right) / W_{0} & 0 \leq k \leq W_{0}-1 \\ P_{l}\{m, k \mid m, 0\}=\left(p_{l, 1}+p_{l, 2}\right) / W_{m} & 0 \leq k \leq W_{m}-1\end{cases}
$$

$p_{l, 1}$ and $p_{l, 2}$ in (23) depend on the access types.

For basic access, 


$$
\begin{aligned}
& p_{l, 1}=\left(1-\left(1-p_{l, c}\right) \cdot\left(1-p_{l, e}^{H}\right)\right)+\left(1-p_{l, c}\right) \cdot\left(1-p_{l, e}^{H}\right) \cdot\left(p_{l, e}^{B} \cdot p_{l, e}^{N A K}+\left(1-p_{l, e}^{B}\right) \cdot p_{l, e}^{A C K}\right) \\
& p_{l, 2}=\left(1-p_{l, c}\right) \cdot\left(1-p_{l, e}^{H}\right) \cdot p_{l, e}^{B} \cdot\left(1-p_{l, e}^{N A K}\right)
\end{aligned}
$$

For RTS/CTS access,

$$
\begin{aligned}
& p_{l, 1}=1-\left(1-p_{l, c}\right)\left(1-p_{l, e}^{R T S}\right)\left(1-p_{l, e}^{C T S}\right) \\
& p_{l, 2}=\left(1-p_{l, c}\right)\left(1-p_{l, e}^{R T S}\right)\left(1-p_{l, e}^{C T S}\right)\left(1-\left(1-p_{l, e}^{D A T A}\right)\left(1-p_{l, e}^{A C K}\right)\right)
\end{aligned}
$$

The relations among the stationary distributions of the Markov chain states are

$$
\begin{cases}b_{l, m, k}=\frac{W_{m}-k}{W_{m}} \times\left(b_{l, m-1,0} \cdot p_{l, 1}+b_{l, m, 0} \cdot\left(p_{l, 1}+p_{l, 2}\right)\right) & 0 \leq k \leq W_{m}-1 \\ b_{l, i, k}=\frac{W_{i}-k}{W_{i}} \times\left(b_{l, i-1,0} \cdot p_{l, 1}+b_{l, i+1,0} \cdot\left(1-p_{l, 1}-p_{l, 2}\right)+b_{l, i, 0} \cdot p_{l, 2}\right) & 1 \leq i \leq m-1,0 \leq k \leq W_{i}-1 \\ b_{l, 0, k}=\frac{W_{0}-k}{W_{0}} \times\left(b_{l, 0,0} \cdot\left(1-p_{l, 1}\right)+b_{l, 1,0} \cdot\left(1-p_{l, 1}-p_{l, 2}\right)\right) & 0 \leq k \leq W_{0}-1\end{cases}
$$

From (28), we get

$$
\left\{\begin{array}{l}
b_{l, 1,0}=b_{l, 0,0} \frac{p_{l, 1}}{1-p_{l, 1}-p_{l, 2}} \\
b_{l, i, 0}=\frac{b_{l, i-1,0} \cdot\left(1-p_{l, 2}\right)-b_{l, i-2,0} \cdot p_{l, 1}}{1-p_{l, 1}-p_{l, 2}} \quad 2 \leq i \leq m-1 \\
b_{l, m, 0}=\frac{b_{l, m-1,0} \cdot p_{l, 1}}{1-p_{l, 1}-p_{l, 2}}
\end{array}\right.
$$

Based on (29), all $b_{l, i, 0}(1 \leq i \leq m)$ can be expressed as functions of $b_{l, 0,0}$ iteratively.

From (11), (12), (13), (28) and (29), $b_{l, 0,0}, \tau_{l}$, and $p_{l, c}$ can be solved numerically. Then, from (14), the throughput over link type $I$ measured by MAC layer payload, $S_{l}$, can be derived.

\section{3). Backoff-4 Algorithm + IR Scheme for Heterogeneous Links}

In the following analysis, we consider the case that the "maximum number of immediate retries" in IR scheme is 1 , that is, a data frame that suffers from a transmission loss will be retransmitted immediately only once. If this retry fails, a backoff is performed. For RTS/CTS access, IR scheme retransmits the data frame only. When the IR scheme is integrated to Backoff- 4 algorithm, the state 
transition diagram is not changed (Figure 4). The analytical procedure is basically the same as that for the Backoff-4 algorithm except for the following modifications.

The only non-null one-step transition probabilities are still given by (23), but $p_{l, 2}$ needs to be redefined. For basic access,

$$
p_{l, 2}=\left(1-p_{l, c}\right) \cdot\left(1-p_{l, e}^{H}\right) \cdot p_{l, e}^{B} \cdot\left(1-p_{l, e}^{N A K}\right) \cdot\left(1-\left(1-p_{l, e}^{D A T A}\right)\left(1-p_{l, e}^{A C K}\right)\right)
$$

For RTS/CTS access,

$$
p_{l, 2}=\left(1-p_{l, c}\right)\left(1-p_{l, e}^{R T S}\right)\left(1-p_{l, e}^{C T S}\right)\left(1-\left(1-p_{l, e}^{D A T A}\right)\left(1-p_{l, e}^{A C K}\right)\right)^{2}
$$

Using the same numerical method as that for the Backoff-4 algorithm, $b_{l, 0,0}, \tau_{l}$, and $p_{l, c}$ can be solved. Then, the throughput over link type $l, S_{l}$, is given by

$$
S_{l}=\frac{p_{l, s}^{*} p_{t r} D}{\left(1-p_{t r}\right) \sigma+p_{t r} T^{*}}
$$

For basic access,

$$
\begin{aligned}
& p_{l, s}^{*}=p_{l, s}^{* \text { basic }}=\left(1-\left(1-\left(1-p_{l, e}^{\text {DATA }}\right) \cdot\left(1-p_{l, e}^{A C K}\right)\right)^{2}\right) \cdot p_{l, g} \\
& T^{*}=T^{* \text { basic }}=T^{\text {basic }}+\left(T_{\text {SIFS }}+T^{\text {basic }}\right) \cdot \sum_{l=1}^{L}\left(\left(1-\left(1-p_{l, e}^{\text {DATA }}\right) \cdot\left(1-p_{l, e}^{A C K}\right)\right) \cdot p_{l, g}\right)
\end{aligned}
$$

For RTS/CTS access,

$$
\begin{aligned}
& p_{l, s}^{*}=p_{l, s}^{* R C T S}= \\
& \left(1-\left(1-\left(1-p_{l, e}^{R T S}\right) \cdot\left(1-p_{l, e}^{C T S}\right) \cdot\left(1-p_{l, e}^{D A T A}\right) \cdot\left(1-p_{l, e}^{A C K}\right)\right) \cdot\left(1-\left(1-p_{l, e}^{D A T A}\right) \cdot\left(1-p_{l, e}^{A C K}\right)\right)\right) \cdot p_{l, g} \\
& T^{*}=T^{* R C T S}=T^{R C T S}+ \\
& \left(T_{D I F S}+T_{D A T A}+T_{S I F S}+T_{A C K}\right) \cdot \sum_{l=1}^{L}\left(\left(1-p_{l, e}^{R T S}\right) \cdot\left(1-p_{l, e}^{C T S}\right) \cdot\left(1-\left(1-p_{l, e}^{D A T A}\right) \cdot\left(1-p_{l, e}^{A C K}\right)\right) \cdot p_{l, g}\right)
\end{aligned}
$$

Other definitions in (32) are same as those in (14).

\section{Numerical Results and Discussions}

In this section, we present numerical results based on the above theoretical analyses. 802.11b physical layer is examined. The default parameters of $802.11 \mathrm{~b}$ are given in Table 2. We have also 
examined 802.11b with other possible parameter values (e.g., different CWmin values), and 802.11a/11g standards. As the conclusions are similar, they are not presented here due to space limitation. All analytical results have been verified by NS-2 [23] simulations. Again due to space limitation, only some simulation results are presented here.

We use the method given in the IEEE standards [18][19][20] to calculate the bit error rates (BERs) and frame error rates (FERs) in a WLAN. This method has also been used in [15]$[17][21][22]$ to study WLAN performance. It is briefly described as follows.

First, the symbol error rate (SER) is calculated based on the SINR at the receiver. It is assumed that the interference and noise affect the desired signal in a manner equivalent to additive white Gaussian noise (AWGN). Given the number of bits per symbol, the SER is then converted into an effective BER. IEEE 802.11b uses complementary code keying (CCK) modulation to achieve its higher data rates (5.5 Mbps and $11 \mathrm{Mbps)}$ [6]. The SER in CCK [10][18][20] has been determined as

$$
S E R=\sum Q \sqrt{2 \times S I N R \times R_{c} \times D_{c}}
$$

where, $R_{c}$ is the code rate, $D_{c}$ is the codeword distance, and, $\sum$ is over all codewords.

For 11 Mbps data rate, the SER is given by

$$
\begin{gathered}
S E R_{11}=24 \times Q(\sqrt{4 \times S I N R})+16 \times Q(\sqrt{6 \times S I N R})+174 \times Q(\sqrt{8 \times S I N R})+ \\
16 \times Q(\sqrt{10 \times S I N R})+24 \times Q(\sqrt{12 \times S I N R})+Q(\sqrt{16 \times S I N R})
\end{gathered}
$$

As each symbol encodes 8 bits in 11 Mbps, the BER is

$$
B E R_{11}=\frac{2^{8-1}}{2^{8}-1} \times S E R_{11}=\frac{128}{255} \times S E R_{11}
$$

When the BERs have been determined, the FERs of the data and control frames are derived from the BERs and the frame lengths.

\section{1) Loss Differentiation Probabilities}

First we evaluate the effectiveness of the LD methods for small (150 bytes) and large (1500 bytes) data frame payloads. The numerical results in Table 3 show that the probabilities of successful 
transmission loss detection under various link conditions are high. The probabilities of successful detection for RTS/CTS access are higher than 97\% regardless of the payload size. The probabilities of successful detection for basic access are about $80 \%$ for small payload and better than $95 \%$ for large payload, respectively. Therefore the proposed LD methods are highly effective. Note that in Table 3, DataRate (for data frame transmissions) and BasicRate (for control frame transmissions) are assigned the same value. If BasicRate < DataRate, as is normally the case in a practical WLAN, the probability of successful transmission loss detection can be even higher.

\section{2) Throughputs of the Backoff Algorithms under Homogeneous Link Condition}

Next we examine the throughputs of the backoff algorithms under homogenous link condition; i.e., all transmissions experience the same SINR over the wireless links. Figure 5 and Figure 6 show the performance comparisons of the backoff algorithms for basic and RTS/CTS access, respectively, with a MAC payload size of 1000 bytes, and receive $\operatorname{SINR}=6.7 \mathrm{~dB}$ so that $B E R_{11}=10^{-4}$ by (39).

It can be seen that the standard backoff algorithm (Backoff-1) produces poor performance, due to incorrect doubling of backoff time when transmission losses occur. Although [2][3][4] reveal that Backoff-2 performs better than Backoff-1 when there are many stations accessing an error-free channel, the opposite is true when the channel is noisy and prone to errors. Without LD, the contention window can unnecessarily reach a large value because of frame errors. In Case- 2 in Table 1, if the contention window is halved instead of immediately going back to CWmin as in the standard, the air time in Backoff-2 can be more severely wasted.

Backoff-3 and Backoff-4 enhanced with LD capability both significantly outperform the existing Backoff-1 and Backoff-2 algorithms. When the number of stations is small, there is no obvious performance difference between Backoff-3 and Backoff-4. However, when there are many stations, Backoff-4 outperforms Backoff-3. The performance difference can be more substantial when the BER over the wireless link is low. In the case when the BER is 0, Backoff-3 behaves exactly the same as Backoff-1, and Backoff-4 as Backoff-2. As shown in [2][3][4], Backoff-2 
significantly outperforms Backoff-1 in this case. In the same way, Backoff-4 outperforms Backoff-3 and Backoff-1. Therefore Backoff-4 is the most desirable algorithm among the four.

When the IR scheme is applied to Backoff-4, more throughput improvement can be obtained due to the reduced contention and the increased transmission success probability.

The simulation results are also given in Figure 5 and Figure 6 to verify the correctness of the theoretical analysis. It can be seen that the theoretical analysis is accurate.

In a practical WLAN environment, the number of active stations generating saturated traffic at any time may not be large. Figure 7 gives the throughputs and throughput ratios between Backoff-4 + IR and Backoff-1 over a range of BER values when there is only one station. It can be seen that when BER $>10^{-4}$, about $100 \%$ throughput improvement can be obtained for both basic and RTS/CTS access methods.

\section{3) Throughputs and Fairness of the Backoff Algorithms under Heterogeneous Link}

\section{Conditions}

In a WLAN environment with heterogeneous wireless link conditions, fairness is an important issue. As will be shown, there is a severe unfairness problem with the standard backoff algorithm in this case.

There are two popular definitions of fairness: max-min fairness [24] and proportional fairness [25]. Max-min fairness tries to give all participants equal throughput while proportional fairness tries to maximize the overall utility of throughputs.

Consider the case in which there are two types of wireless links with different SINR values, "good" link (the FER of data frames caused by noise is 0.1) and "bad" link (the FER of data frames caused by noise is 0.5), in a WLAN. The MAC payload size of the data frame is again 1000 bytes. A station in the WLAN operates on an either "good" or "bad" link. As we consider the same number of "good" and "bad" stations (n), the proportional fairness utility (PFU) is defined as

$$
P F U=\sum_{i=1}^{n} S_{g, i} \times \sum_{i=1}^{n} S_{b, i}
$$


where $S_{g, i}$ is the throughput (in Mbps) of the ith "good" station and $S_{b, i}$ the throughput of the ith “bad” station. A larger PFU value indicates better fairness. The throughputs and the proportional fairness utilities of the two types of stations are compared in Figure 8 (basic access) and Figure 9 (RTS/CTS access).

The standard backoff algorithm (Backoff-1) produces the worst fairness because the backoff time is always doubled even a data frame is lost due to noise. The stations experiencing a "bad" link keep the contention window at a higher level and therefore have less opportunity to send data. In contrast, the stations experiencing a "good" link not only have a higher probability to transmit their data frames successfully, but also have more access opportunity because of their smaller contention windows. The incorrect backoff procedure leads to the unfairness.

By introducing the LD capability and modifying the backoff procedure accordingly, fairness can be significantly improved. Figure 8 and Figure 9 show that in terms of both max-min and proportional fairness definitions, Backoff-4 outperforms the standard backoff. The IR scheme bundled with Backoff-4 can further improve the fairness especially when there are a large number of stations.

\section{CONCLUSIONS}

Data frame transmissions over a WLAN may suffer from transmission losses as well as collision losses. The standard backoff algorithm in the IEEE 802.11 DCF does not distinguish between these two types of frame losses, and incorrectly doubles the contention window when transmission losses occur. To overcome this shortcoming, this paper proposes new backoff algorithms based on loss differentiation capability, and presents extensive performance evaluations to demonstrate the effectiveness of the proposed methods. One of these methods, called Backoff-4, is recommended due to the following advantages: (i) simple implementation that requires minimal modification to the standard; (ii) negligible overhead; and, (iii) substantial improvements in system throughput and fairness. 
To the best of our knowledge, this paper is the first to show the undesirable behavior of the standard backoff procedure when transmission losses occur, to develop a practical solution to this problem, and to give a theoretical performance analysis of different backoff algorithms under heterogeneous link conditions.

One future work is to investigate the possibility of integrating the loss differentiation methods with those on-line dynamic contention window adjustment schemes such as the one in [26]. Another interesting future work is how to adapt the new backoff schemes discussed in this paper into the new 802.11n and 802.11e [12] standards for next-generation WLANs.

\section{REFERENCES}

[1] IEEE Std 802.11-1999 (R2003), Wireless Lan Medium Access Control (MAC) and Physical Layer (PHY) Specifications, Jun. 2003.

[2] H. Wu, S. Cheng, Y. Peng, K. Long, and J. Ma, "IEEE 802.11 distributed coordination function (DCF): analysis and enhancement,” IEEE ICC 2002, pp.605-609, Apr.-May 2002.

[3] N. Song, B. Kwak, J. Song, and L.E. Miller, "Enhancement of IEEE 802.11 distributed coordination function with exponential increase exponential decrease backoff algorithm,” IEEE VTC 2003 Spring, pp.2775-2778, Apr. 2003.

[4] Q. Pang, S.C. Liew, J. Lee, and V. Leung, "Performance evaluation of an adaptive backoff scheme for WLAN,” Wireless Communications and Mobile Computing, vol.4, no.8, pp. 867879, Dec. 2004.

[5] Q. Pang, S.C. Liew, and V. Leung, "Design of an effective loss-distinguishable MAC protocol for 802.11 WLAN,” IEEE Communications Letters, vol. 9, no. 9, pp.781-783, Sep. 2005.

[6] IEEE Std 802.11b-1999, Wireless LAN Medium Access Control (MAC) and Physical Layer (PHY) Specifications: Higher-speed Physical Layer Extension In The 2.4 GHz Band, Sep. 1999.

[7] IEEE Std 802.11g-2003, Wireless LAN Medium Access Control (MAC) and Physical Layer (PHY) Specifications Amendment 4: Further Higher Data Rate Extension in the $2.4 \mathrm{GHz}$ Band, June 2003.

[8] ATM Forum, ATM User-Network Interface Specification V3.1, Sep. 1994.

[9] IEEE Std 802.15.1, Wireless Medium Access Control (MAC) and Physical Layer (PHY) Specifications for Wireless Personal Area Networks (WPANs), Jun. 2002.

[10] WiMedia Alliance, URL http://www.wimedia.org. 
[11] G. Chesson, W. Diepstraten, D. Kitchin, H. Teunissen, and M. Wentink, "Baseline D-QoS proposal,” IEEE 802.11 working document 802.11-00/399, Nov. 2000.

[12] IEEE Std 802.11e-2005, Wireless LAN Medium Access Control (MAC) and Physical Layer (PHY) Specifications Amendment 8: Medium Access Control (MAC) Quality of Service Enhancements, Nov. 2005.

[13] G. Bianchi, "Performance analysis of the IEEE 802.11 distributed coordination function," IEEE J. Select. Areas Commun., vol.18, no.3, pp.535-547, Mar. 2000.

[14] Y. C. Tay and K. C. Chua, “A capacity analysis for the IEEE 802.11 MAC protocol," Wireless Networks, vol.7, no.2, pp.159-171, 2001.

[15] P. Chatzimisios, A.C. Boucouvalas, and V. Vitsas, "Performance analysis of IEEE 802.11 DCF in presence of transmission errors,” IEEE ICC 2004, pp. 3854-3858, Jun. 2004.

[16] J. Yeo and A. Agrawala, "Packet error model for the IEEE 802.11 MAC protocol," IEEE PIMRC 2003, pp.1722-1726, Jan. 2003.

[17] J. Yin, X. Wang, and D.P. Agrawal, “Optimal packet size in error-prone channel for IEEE 802.11 distributed coordination function,” IEEE WCNC 2004, pp.1654-1659, Mar. 2004.

[18] IEEE Std 802.15.2, Coexistence of Wireless Personal Area Networks with Other Wireless Devices Operating in Unlicensed Frequency Bands, Aug. 2003.

[19] IEEE Std 802.15.3, Wireless Medium Access Control (MAC) and Physical Layer (PHY) Specifications for High Rate Wireless Personal Area Networks (WPANs), Sep. 2003.

[20] IEEE Std 802.15.4, Wireless Medium Access Control (MAC) and Physical Layer (PHY) Specifications for Low-Rate Wireless Personal Area Networks (LR-WPANs), Oct. 2003.

[21] G. Holland, N. Vaidya, and P. Bahl, “A rate-adaptive MAC protocol for multi-hop wireless networks,” ACM MOBICOM 2001, pp.236-250, Jul. 2001

[22] D. Qiao, S. Choi, and K.G. Shin, "Goodput analysis and link adaptation for IEEE 802.11a wireless LANs,” IEEE Transactions on Mobile Computing, vol.1 no. 4, pp.278-292, Oct.-Dec. 2002.

[23] NS-2, URL http://www.isi.edu/nsnam/ns.

[24] D. Bertsekas and R. Gallager, Data Networks, Prentice Hall, 1992.

[25] F. Kelly, "Charging and rate control for elastic traffic,” Eur. Trans. Telecommun., vol.8, pp.237-252, 1997.

[26] G. Bianchi and I. Tinnirello, "Kalman filter estimation of the number of competing terminals in an IEEE 802.11 network,” IEEE INFOCOM 2003, pp.844-852. Mar.-Apr. 2003. 


\section{List of Figures}

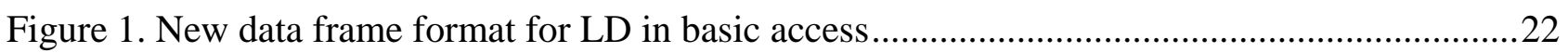

Figure 2. Flow charts for LD in basic access at receiver and sender ...............................................22

Figure 3. State transition diagram of the standard backoff algorithm over link type $l$......................23

Figure 4. State transition diagram of Backoff-4 algorithm over link type 1 .......................................24

Figure 5. Comparison of the backoff algorithms for basic access .....................................................25

Figure 6. Comparison of the backoff algorithms for RTS/CTS access.............................................25

Figure 7. Throughput under different wireless link conditions for a single accessing station............26

Figure 8. Comparison of the backoff algorithms under heterogeneous conditions for basic access .27

Figure 9. Comparison of the backoff algorithms under heterogeneous conditions for RTS/CTS access.

\section{List of Tables}

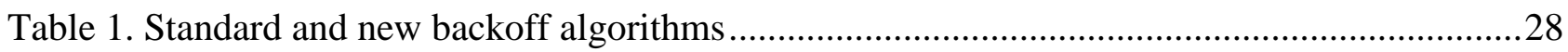

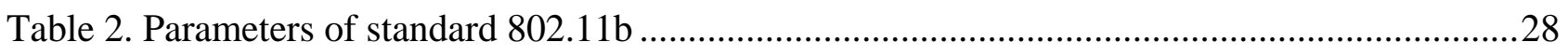

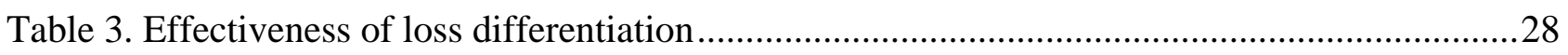




\begin{tabular}{|c|c|c|c|}
\hline $18 \sim 30$ & $1 \sim 2$ & $0 \sim 2312$ & 4 \\
\hline Frame control/Duration/Addresses & HEC & Frame Body & FCS \\
\hline
\end{tabular}

Figure 1. New data frame format for LD in basic access

(a) RECEIVER

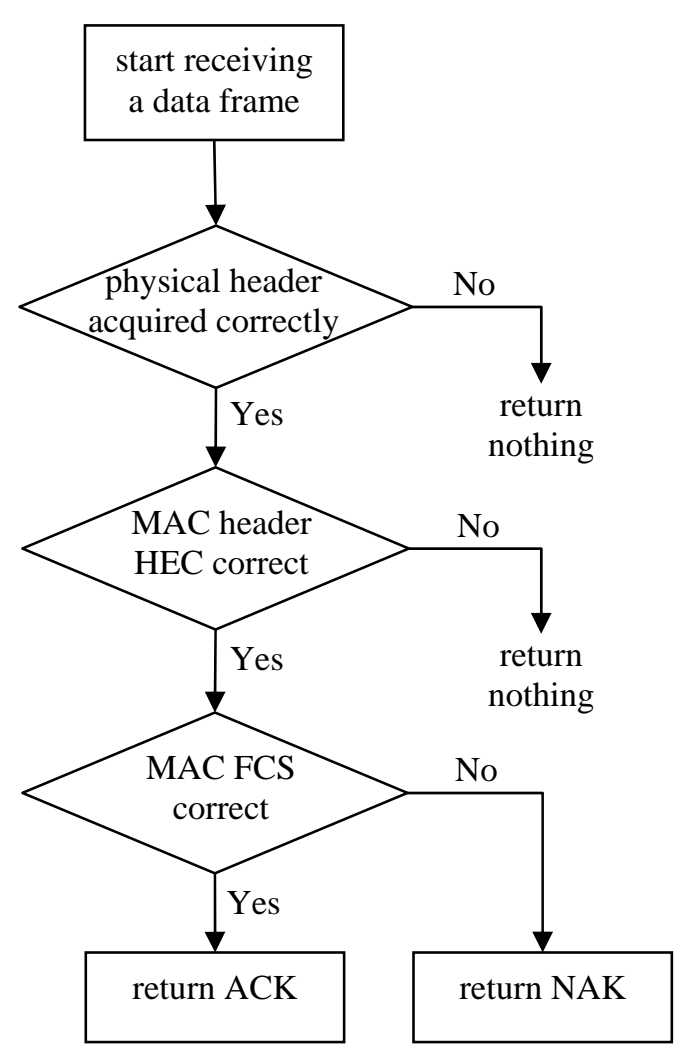

(b) SENDER

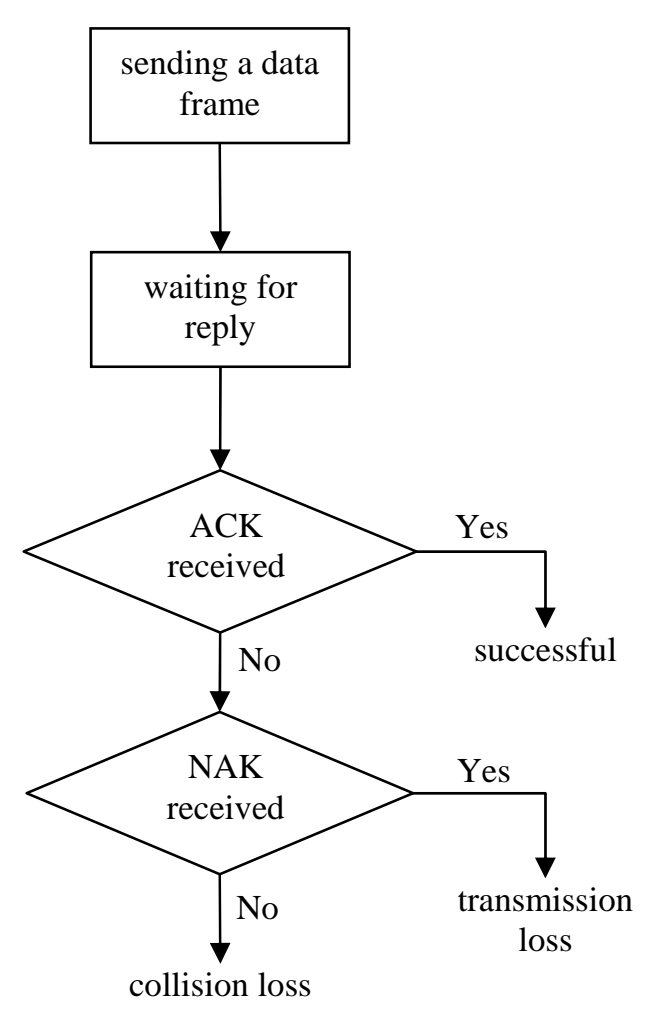

Figure 2. Flow charts for $\mathrm{LD}$ in basic access at receiver and sender 


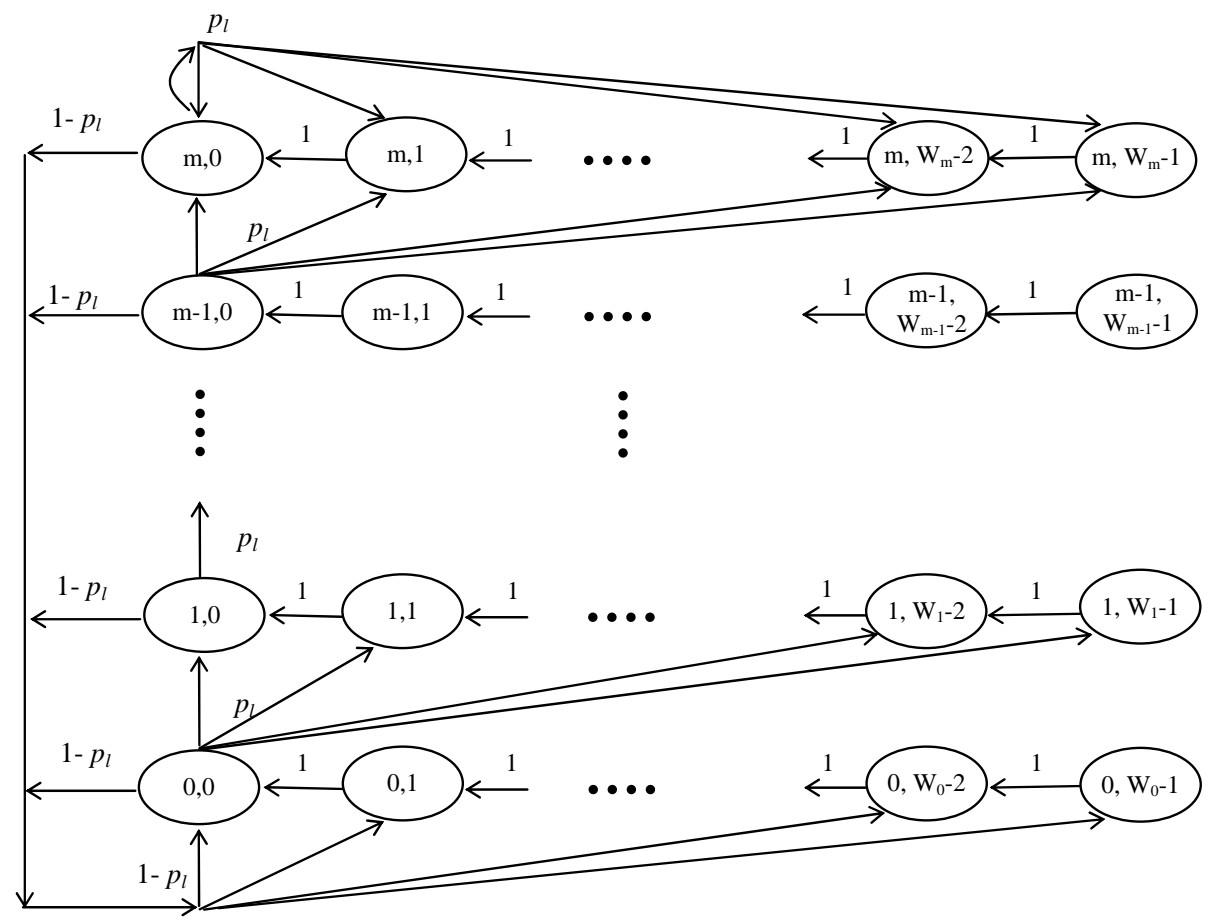

Figure 3. State transition diagram of the standard backoff algorithm over link type $l$ 


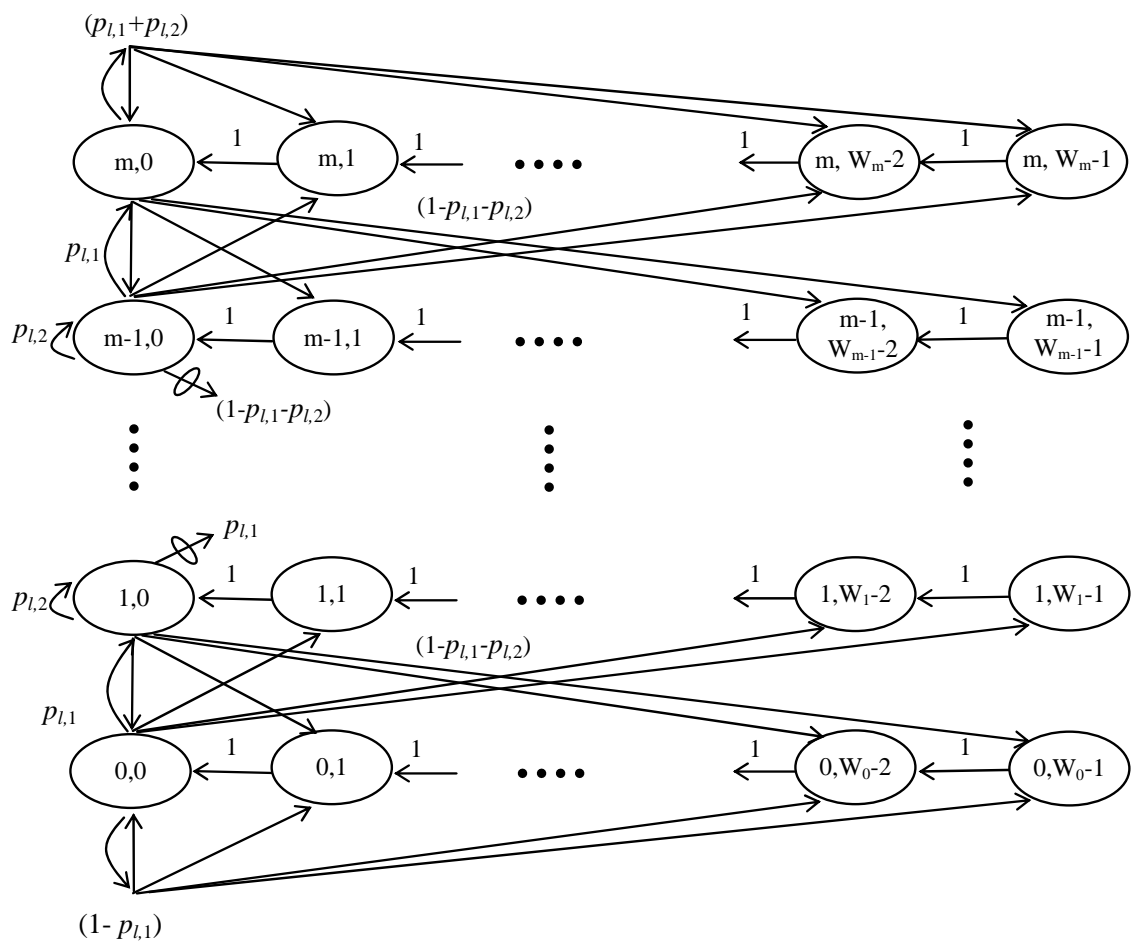

Figure 4. State transition diagram of Backoff-4 algorithm over link type $l$ 


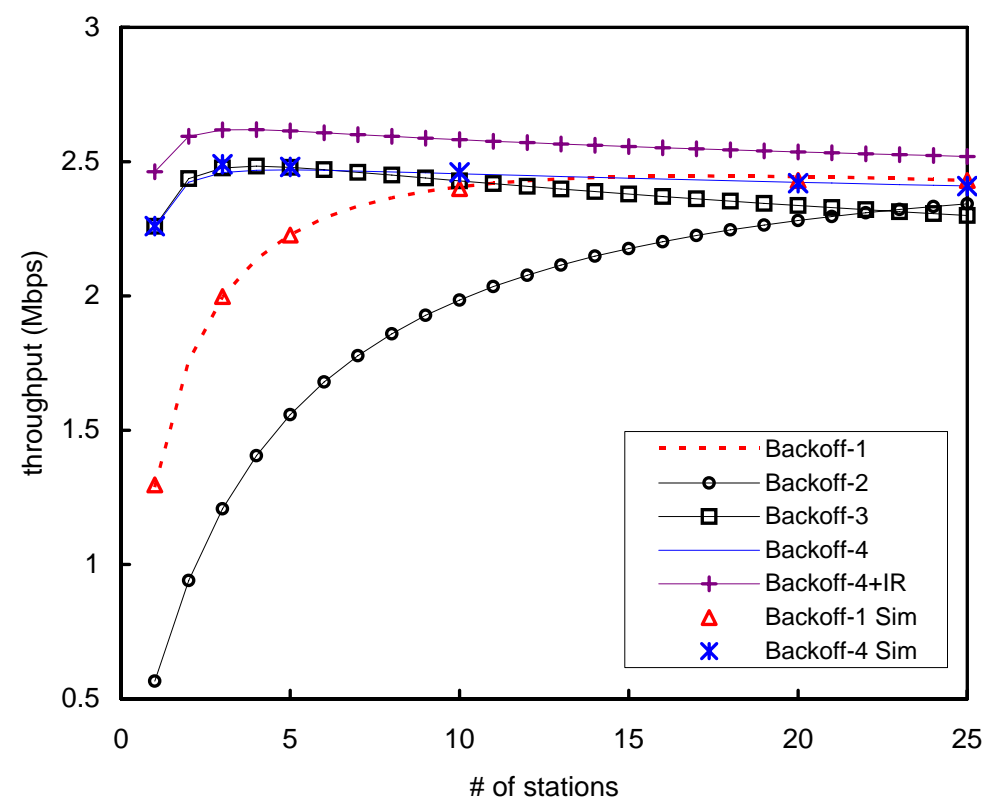

Figure 5. Comparison of the backoff algorithms for basic access

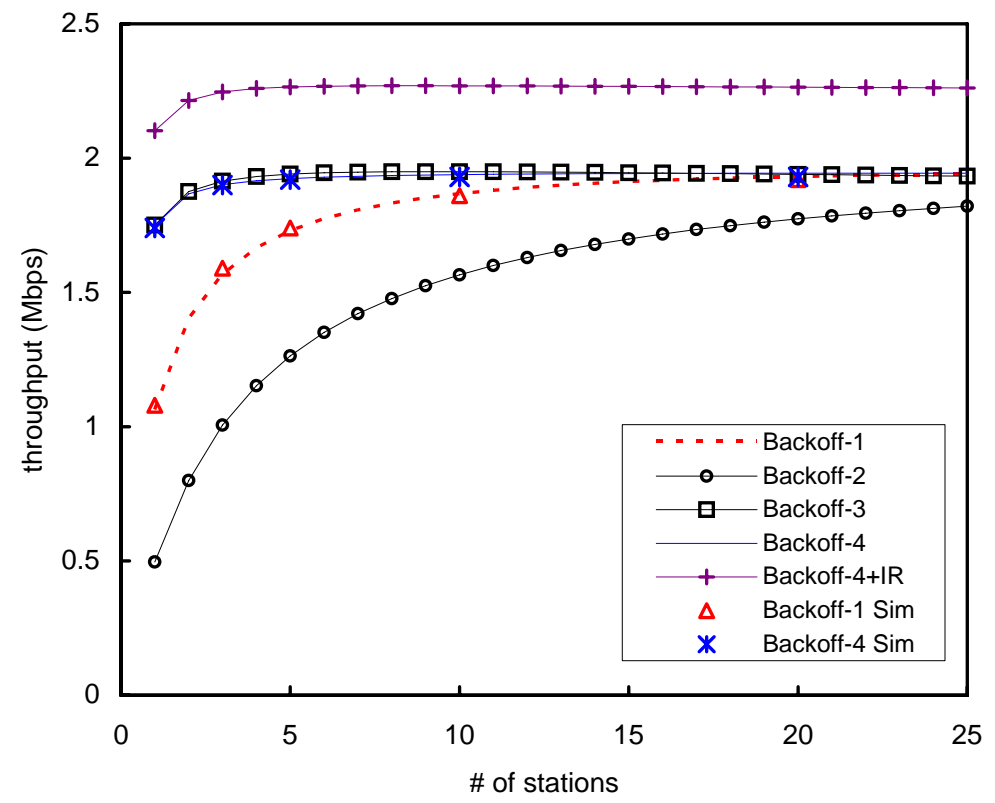

Figure 6. Comparison of the backoff algorithms for RTS/CTS access 


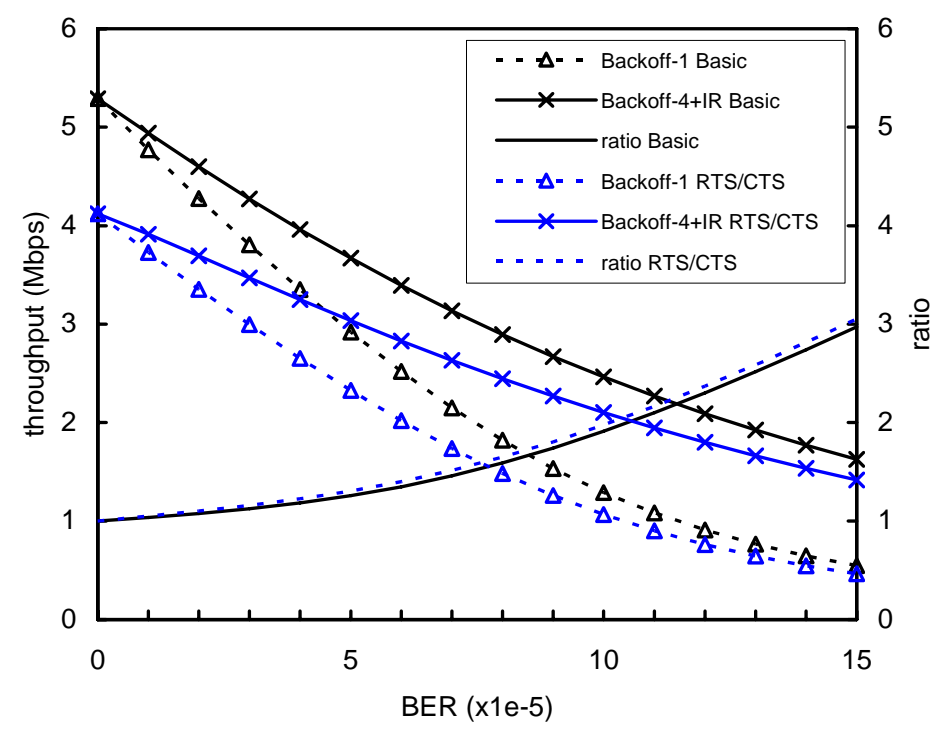

Figure 7. Throughput under different wireless link conditions for a single accessing station 

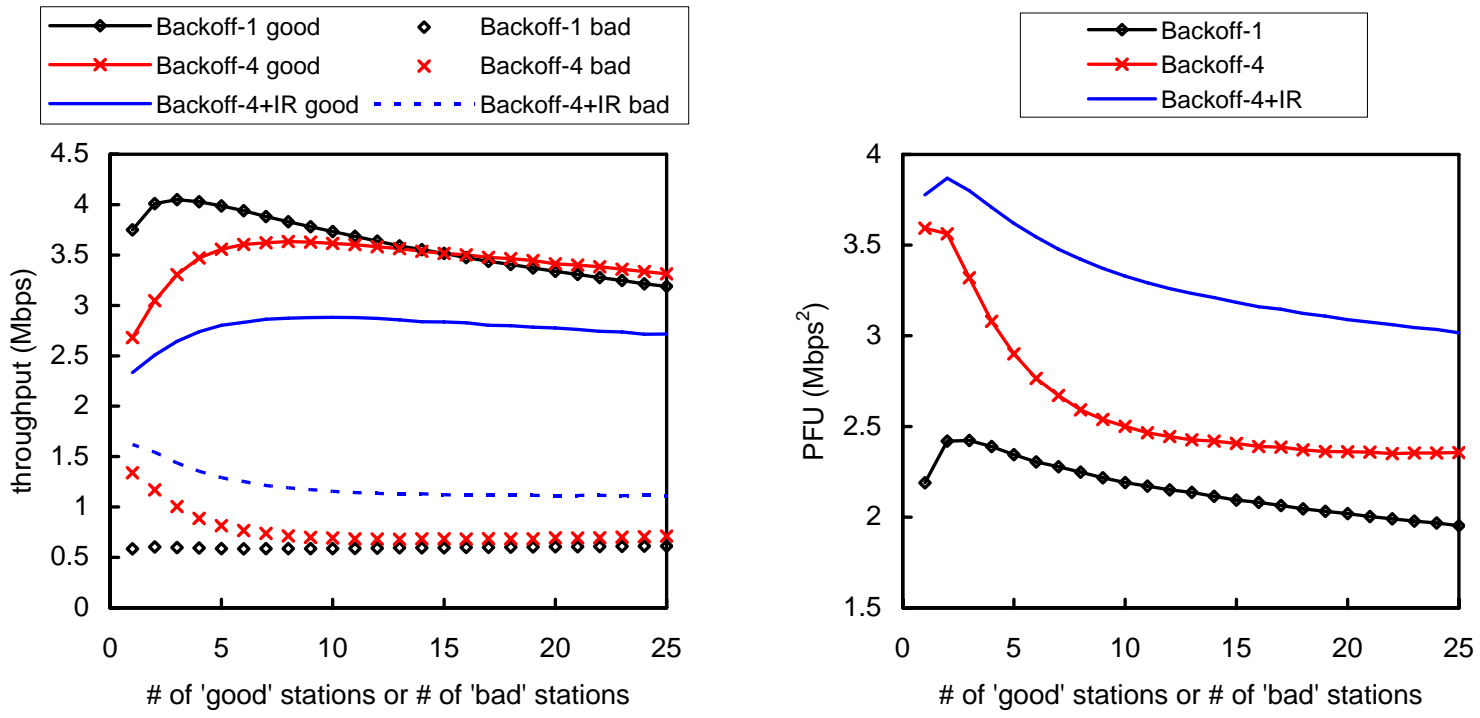

Figure 8. Comparison of the backoff algorithms under heterogeneous conditions for basic access
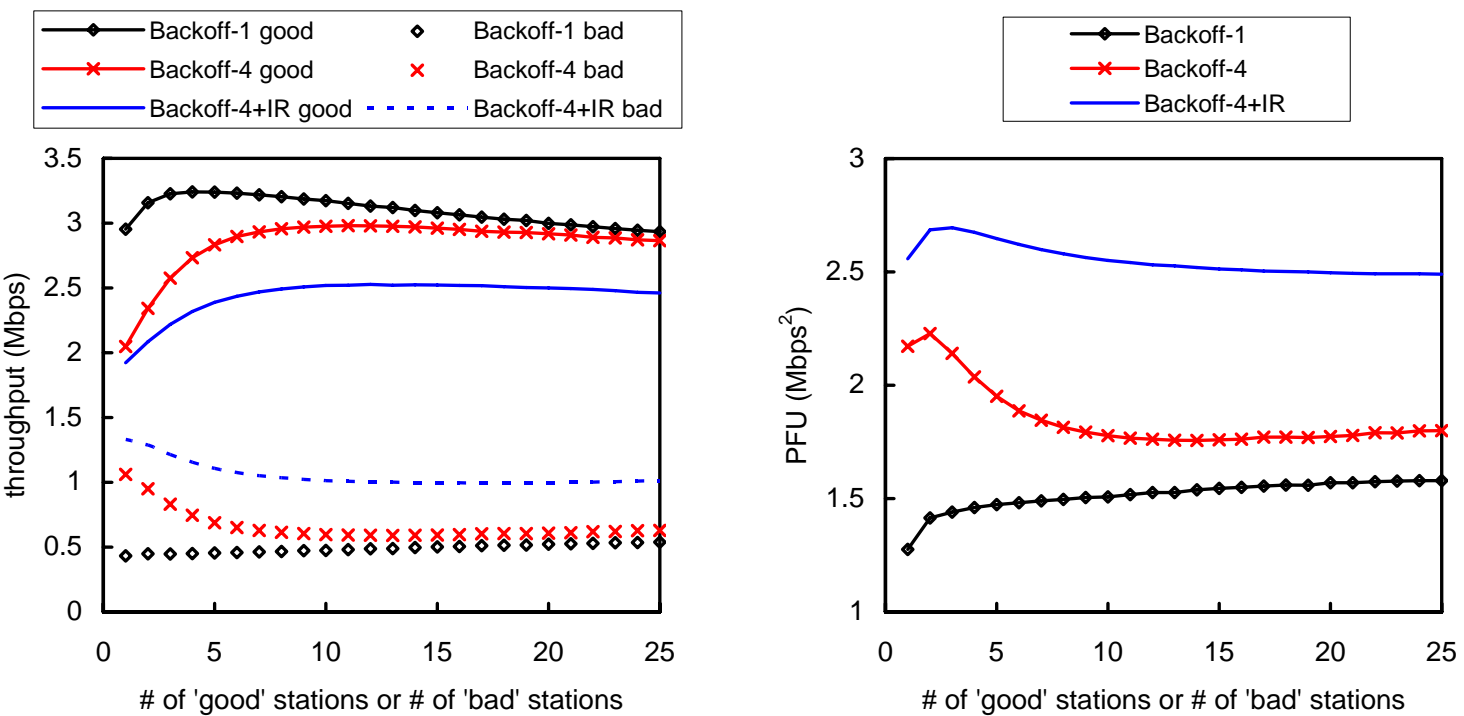

Figure 9. Comparison of the backoff algorithms under heterogeneous conditions for RTS/CTS access 
Table 1. Standard and new backoff algorithms

\begin{tabular}{|c|c|c|c|}
\hline & $\begin{array}{c}\text { Case-1: } \\
\text { a collision occurs }\end{array}$ & $\begin{array}{c}\text { Case-2: } \\
\text { transmission is in-error due } \\
\text { to noise }\end{array}$ & $\begin{array}{c}\text { Case-3: } \\
\text { transmission is successful }\end{array}$ \\
\hline $\begin{array}{c}\text { Backoff-1 } \\
\text { (standard) }\end{array}$ & $c w \leftarrow \min \{c w \times 2, C W \max \}$ & $c w \leftarrow \min \{c w \times 2, C W \max \}$ & $c w \leftarrow C W \min$ \\
\hline Backoff-2 & $c w \leftarrow \min \{c w \times 2, C W \max \}$ & $c w \leftarrow \min \{c w \times 2, C W m a x\}$ & $c w \leftarrow \max \{c w / 2, C W \min \}$ \\
\hline Backoff-3 & $c w \leftarrow \min \{c w \times 2, C W \max \}$ & $c w \leftarrow c w$ & $c w \leftarrow C W \min$ \\
\hline Backoff-4 & $c w \leftarrow \min \{c w \times 2, C W \max \}$ & $c w \leftarrow c w$ & $c w \leftarrow \max \{c w / 2, C W \min \}$ \\
\hline
\end{tabular}

Table 2. Parameters of standard 802.11b

\begin{tabular}{|c|c|}
\hline CWmin & 32 \\
\hline CWmax & 1024 \\
\hline SlotTime & $20 \mu \mathrm{s}$ \\
\hline CCATime & $15 \mu \mathrm{s}$ \\
\hline RxTxTurnaroundTime & $5 \mu \mathrm{s}$ \\
\hline PHY overhead & $192 \mu \mathrm{s}$ \\
\hline DataRate & $11 \mathrm{Mbps}$ \\
\hline BasicRate & $11 \mathrm{Mbps}$ \\
\hline SIFS & $10 \mu \mathrm{s}$ \\
\hline DIFS & $50 \mu \mathrm{s}$ \\
\hline data frame payload & 1000 bytes \\
\hline ACK, NAK, CTS frames & 112 bits \\
\hline RTS frame & 160 bits \\
\hline
\end{tabular}

Table 3. Effectiveness of loss differentiation

\begin{tabular}{|c|c|c|c|}
\hline$B E R_{11}$ & $1 \mathrm{e}-5$ & $5 e-5$ & $1 \mathrm{e}-4$ \\
\hline$p_{e}^{R T S}$ & 0.002 & 0.008 & 0.016 \\
\hline$p_{e}^{C T S}, p_{e}^{A C K}, p_{e}^{N A K}$ & 0.001 & 0.006 & 0.011 \\
\hline$p_{e}^{H}$ & 0.002 & 0.010 & 0.019 \\
\hline$p_{e}^{D A T A}$ (small payload) & 0.014 & 0.069 & 0.133 \\
\hline$p_{e}^{D A T A}$ (large payload) & 0.115 & 0.458 & 0.706 \\
\hline$P_{D}^{\text {basic }}$ (small payload) & $80.2 \%$ & $79.7 \%$ & $79.1 \%$ \\
\hline$P_{D}^{\text {basic }}$ (large payload) & $97.4 \%$ & $96.7 \%$ & $95.8 \%$ \\
\hline$P_{D}^{R C T S}$ & $99.7 \%$ & $98.6 \%$ & $97.3 \%$ \\
\hline
\end{tabular}

